\title{
IMPACTO DE LAS ENERGÍAS RENOVABLES EN LOS SISTEMAS DE GENERACIÓN DISTRIBUIDA
}

\section{Margarita Fredesvinda Murillo Manrique}

\section{Resumen}

El presente artículo corresponde al tipo de investigación descriptiva explicativa, desarrollado como una propuesta a los cambios que deberá enfrentar nuestro país respecto de la aplicación de las energías renovables que son necesarias para promover la competitividad del sistema de generación, asegurando la provisión de energía y protección del medio ambiente.

Se describe el escenario actual de fuerte dependencia de los combustibles fósiles y las energías renovables que se utilizan actualmente de manera aislada, para determinar el impacto en los sistemas de generación distribuida; como resultado, se presentan estadísticas de estudios previos desarrollados por expertos en el campo de la bioenergía en los trabajos realizados en los últimos años; así como la información que hemos obtenido durante esta investigación.

Palabras Clave: Energías renovables, generación distribuida, demanda energética, sistemas eléctricos de potencia.

\footnotetext{
Abstract

This article is the type of explanatory descriptive research, developed as a proposal to the changes that our country will have to face in the implementation of renewable energy that are necessary to promote the competitiveness of the generation system, ensuring energy supply and protection the environment.

The current scenario of strong dependence on fossil fuels and renewable energy currently used in isolation to determine the impact on distributed generation systems is described; as a result, statistics from previous studies conducted by experts in the field of bioenergy in the work carried out in recent years are presented; and the information we have obtained during this investigation.
} 
Keywords: Renewable energy, distributed generation, energy demand, electric power systems.

\section{INTRODUCCIÓN}

El Perú es un país privilegiado por la diversidad de recursos energéticos que posee, tanto renovables como no renovables, sin embargo se debe plantear de manera planificada y sustentada, cuál va ser la mejor estrategia para abastecer la demanda energética derivada del crecimiento económico sostenido en los últimos años y que se espera continúe a un ritmo similar en las siguientes décadas. Considerando que la electricidad es actualmente la forma más útil de energía, ya que está presente en todos los sectores de la sociedad gracias a su gran variedad de aplicaciones, esto nos coloca de cara a un escenario con recursos no renovables agotables que demanda su pronto incremento a través del descubrimiento de nuevas reservas. Por lo tanto, en la actualidad, el desarrollo tecnológico y la progresiva implantación de nuevas fuentes de micro y mini generación, unidos a los proceso de liberación del mercado eléctrico en los países desarrollados y las condiciones de respeto al medio ambiente, hacen que no sea esperable una nueva inversión de esta tendencia, en las cuales las redes eléctricas inteligentes ya se vienen implementando con aplicaciones basadas en energías renovables. Es así que, frente al modelo tradicional implantado en las últimas décadas, surge hoy un modelo alternativo en el que la generación de energía se acerca al consumidor tanto física como virtual- mente. Estos nuevos modelos se denominan Generación Distribuida; por lo tanto, la complementariedad entre ambos modelos será la base para el desarrollo de los futuros sistemas eléctricos de potencia. Para afrontar los retos que en esta especialidad ya son una realidad, se ha elaborado este artículo respecto a las energias renovables, componente importante en la Generación Distribuida, con el ánimo de que los futuros profesionales de la electricidad avizoren las nuevas tendencias en generación, transmisión y distribución de la energía eléctrica relacionadas al uso de las tecnologías y cuidado del medio ambiente; las cuales serán las principales tendencias en el futuro, particularmente en nuestro país.

\section{MATERIAL Y MÉTODOS}

Se realizó un análisis respecto de la información de proyectos energéticos relevantes en nuestro país, del cual se presenta el estado del arte respecto a la generación de energía eléctrica y las capacidades de producción que se deberá considerar en un futuro, según el SEIN-COES. A partir de esa información, se establece la importancia de la generación distribuida.

\section{POLÍTICA Y REGULACIÓN DEL SISTEMA ELÉCTRICO EN EL PERÚ}

La Dirección General de Electricidad (DGE), dependiente del Ministe- 
rio de Energía y Minas (MEM), está a cargo del establecimiento de politicas y regulaciones de electricidad y de otorgar concesiones. También es la responsable de elaborar los planes de expansión de la generación y la transmisión y tiene que aprobar los procedimientos pertinentes para el funcionamiento del sistema eléctrico.

El Organismo Supervisor de la Inversión en Energía y Minería (OSINERGMIN), creado en 1996 como OSINERG, desempeña sus funciones en el sector según lo establecido en la Ley de Concesiones Eléctricas (LCE), de 1992, y la Ley para asegurar el desarrollo eficiente de la Generación Eléctrica (Ley de Generación Eficiente), de 2006, entre otras. Además, el OSINERGMIN es el organismo responsable de hacer cumplir las obligaciones fiscales de los licenciatarios, según lo establecido por la ley y su regulación. Por último, es el responsable de controlar que se cumplan las funciones del Comité de Operación Económica del Sistema (COES) y de determinar semestralmente los porcentajes de la participación de las compañías en el mercado.

En el 2000, OSINERG se fusionó con la Comisión de Tarifas Eléctricas (CTE), actualmente denominada Gerencia Adjunta de Regulación Tarifaria (GART). Juntos, están a cargo de fijar las tarifas de generación, transmisión y distribución y las condiciones de ajuste de tarifas para los consumidores finales.

\section{Participantes del Sector}

Dentro del sector eléctrico se llevan a cabo actividades que tienen una re- lación vertical muy estrecha entre sí. En cuanto a su estructura, el sector se encuentra conformado por cinco actores principales:

- Empresas Eléctricas: Estas se encuentran orientadas a las actividades de generación, transmisión y distribución. Actualmente, son 41 las empresas generadoras, 5 empresas de transmisión y 21 empresas de distribución, según cifras del OSINERGMIN.

- Clientes: Pueden ser divididos a su vez en clientes libres y clientes regulados.

- Comité de Operación Económica del Sistema (COES): Se encuentra conformado por los titulares de las centrales de generación y de transmisión que se encuentran interconectadas al sistema nacional. Este organismo tiene como finalidad coordinar las operaciones al mínimo costo, garantizando la seguridad en el abastecimiento. Su labor es de naturaleza técnica.

- Ministerio de Energía y Minas: Es la entidad encargada de la representación del estado peruano a través de la Dirección General de Electricidad. Este organismo cumple con labores normativas y es el responsable del otorgamiento de concesiones y autorizaciones.

- Organismo Supervisor de la Inversión en Energía y Minería (OSINERGMIN): Es la institución encargada de supervisar, regular, fiscalizar y sancionar a las empresas que se desarrollan en el sector eléctrico, hidrocarburos y minero. 


\section{Gráfico $\mathbf{N}^{\circ} 1$. Actores Del Sector Eléctrico Peruano}

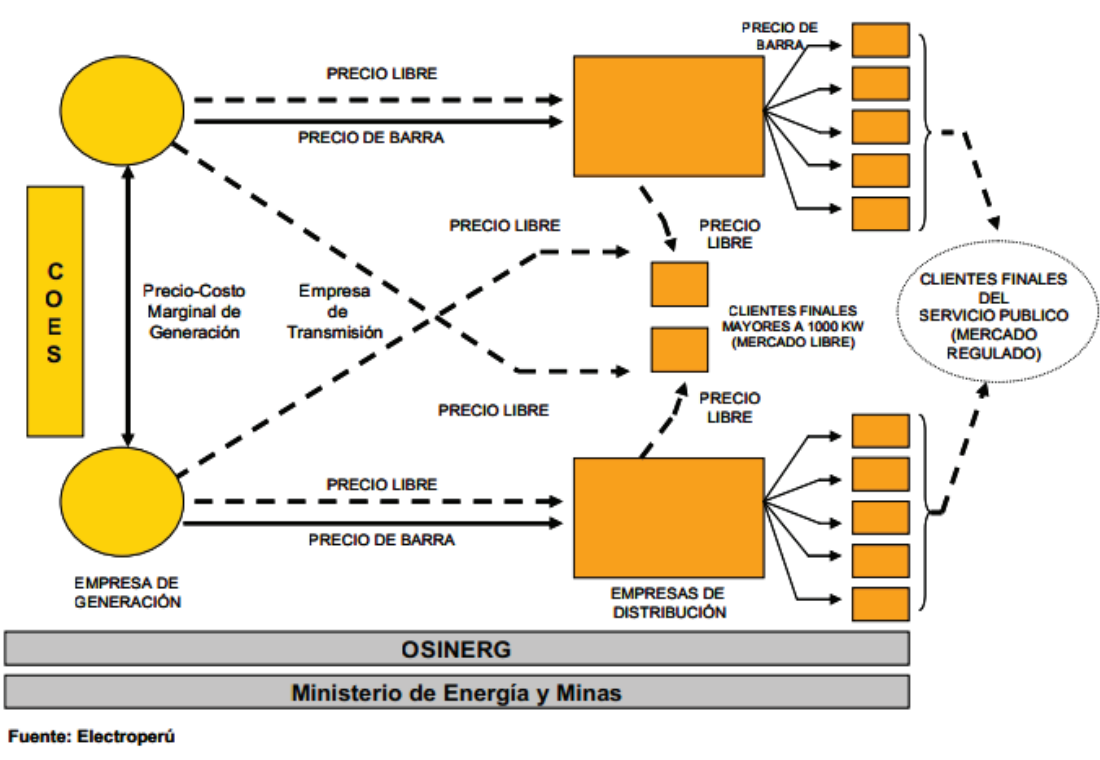

Dependencia de la generación de energia eléctrica

Actualmente, se observa una considerable concentración de generación de energía en el centro del país, específicamente en el departamento de Lima, tanto a nivel de producción como de potencia instalada o efectiva.

Esta concentración, en un mismo espacio geográfico de los niveles de capacidad instalada, puede generar serias complicaciones en el abastecimiento de energía ante cualquier contingencia que se pueda registrar, ya sea natural o de otra indole. Es por ello que se requiere incentivar $\mathrm{y} / \mathrm{o}$ promover la generación en las zonas norte y sur del país, a fin de diversificar la generación de energía, así como acompañar el crecimiento existente de demanda de energía que se viene experimentado en los diferentes departamentos del país, ya que en la actualidad la costa central del país es el principal proveedor de energía a las diferentes regiones, con los problemas que acarrea ello dado el déficit de infraestructura necesaria para atender la creciente demanda.

\section{Cuadro $N^{\circ}$ 1. Demanda de Energia}

\begin{tabular}{|c|c|c||c|c|c||c|c|c|}
\hline Norte & Demanda Total & Nuevos Proyectos & Centro & Demanda Total & Nuevos Proyectos & Sur & Demanda Total & Nuevos Proyectos \\
\hline 2013 & $882 \mathrm{MW}$ & $0 \mathrm{MW}$ & 2013 & $3,993 \mathrm{MW}$ & $251 \mathrm{MW}$ & 2013 & $1,034 \mathrm{MW}$ & $118 \mathrm{MW}$ \\
\hline 2014 & $983 \mathrm{MW}$ & $65 \mathrm{MW}$ & 2014 & $4,310 \mathrm{MW}$ & $405 \mathrm{MW}$ & 2014 & $1,251 \mathrm{MW}$ & $308 \mathrm{MW}$ \\
\hline 2016 & $1,200 \mathrm{MW}$ & $199 \mathrm{MW}$ & 2016 & $4,846 \mathrm{MW}$ & $576 \mathrm{MW}$ & 2016 & $2,106 \mathrm{MW}$ & $1,070 \mathrm{MW}$ \\
\hline 2018 & $1,345 \mathrm{MW}$ & $255 \mathrm{MW}$ & 2018 & $5,503 \mathrm{MW}$ & $714 \mathrm{MW}$ & 2018 & $2,392 \mathrm{MW}$ & $1,283 \mathrm{MW}$ \\
\hline
\end{tabular}




\section{Posible desabastecimiento de ener- gia eléctrica}

En el último informe elaborado por el COES: "Informe de Diagnóstico de las Condiciones Operativas del SEIN 2015-2024", se establece que se podría tener un déficit de generación eficiente del orden de 490 MW entre el 2017 y el 2018, producto del descalce entre la oferta y demanda proyectada, según la información de los proyectos de generación y requerimiento de energía de los diferentes agentes involucrados.

Asimismo, no se cuentan con proyectos de generación posteriores al 2016. Es decir, si en los próximos dos años no se comprometen nuevos proyectos de generación, la confiabilidad del sistema se vería seriamente afectada, considerando las tasas de crecimiento de demanda observadas en los últimos años.
El análisis del COES contempla los escenarios de: i) generación eficiente con proyectos hidroeléctricos únicamente, y ii) generación eficiente con proyectos hidroeléctricos y térmicos. En el primer escenario, es decir, considerando la generación únicamente de las hidroeléctricas, el déficit de generación eficiente ascendería a 980 MW entre el periodo comprendido entre los años 2017 y 2022. No obstante, de considerar las centrales termoeléctricas, el déficit de generación eficiente se reduciria a $490 \mathrm{MW}$ entre dichos años. Es importante mencionar que en este último escenario se toma en consideración plantas de ciclo combinado por 1,500 MW en la macro región sur, asociados al proyecto del Gasoducto Sur Peruano y Nodo Energético en el sur del país.

\section{Gráfico $\mathbf{N}^{\circ}$ 2. Escenarios de Generación en el Sector Eléctrico Peruano}

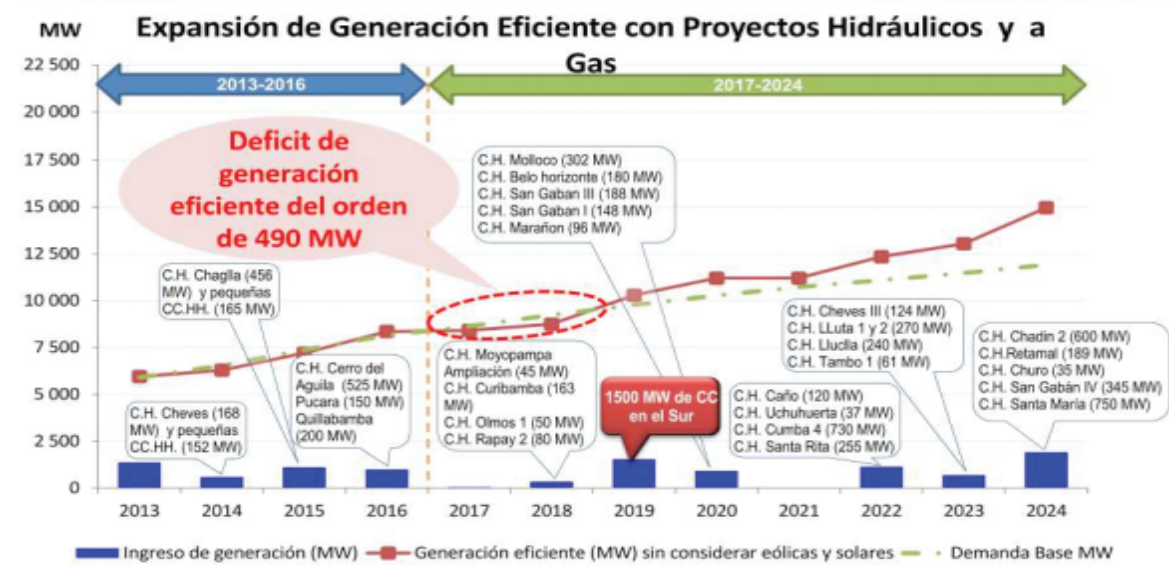

Fuente y elaboración: COES

\section{ENERGÍAS RENOVABLES}

Las energias renovables son aquellas cuyo potencial es inagotable, ya que provienen de la energía que llega a nuestro planeta de forma continua, como consecuencia de la radiación solar o de la atracción gravitatoria de la 
Luna. Son fundamentalmente: la energía hidráulica, la solar, la eólica, la biomasa, la geotérmica y las marinas.

Las energías no renovables son aquellas que existen en la naturaleza en una cantidad limitada. No se renuevan a corto plazo y por eso se agotan cuando se utilizan. La demanda mundial de energía en la actualidad se satisface fundamentalmente con este tipo de fuentes energéticas: el carbón, el petróleo, el gas natural y el uranio. Desde el punto de vista de la utilización de la energía, podemos clasificar la energía en primaria, secundaria y útil.

- Energía primaria: es la que se obtiene directamente de la naturaleza y corresponde a un tipo de energía almacenada o disponible, como por ejemplo, el petróleo, el carbón, el gas natural, el uranio y las energías renovables.

- Energía secundaria (también conocida como energía final): se obtiene a partir de transformaciones de la energía primaria. Ejemplos de esta categoría son la electricidad y la gasolina.

- Energía útil: es la que obtiene el consumidor después de la última conversión realizada por sus propios equipos de demanda, como por ejemplo, la energía mecánica gastada en un motor, la luminosa en una bombilla, etc. Algunas energías primarias pasan directamente a energía útil, sin transformarse previamente en energía secundaria.

Durante los últimos años se ha fomentado la utilización de las energías renovables, que podrian sustituir en gran medida a las convencionales, reduciendo de este modo la dependencia energética y aumentando la autonomía del país. Los diferentes estudios energéticos realizados han dado como resultado altos valores de potencial de energías renovables; ello significa que se podría garantizar una autonomía energética mucho mayor de la actual (a finales de 2012 el porcentaje de electricidad de origen renovable en el Perú rondaba el 4\%).

\section{El agotamiento de los combustibles fósiles}

El sistema energético actual está fundamentalmente basado en los combustibles fósiles. El ritmo de consumo es tal que en un año la humanidad consume lo que la naturaleza tarda un millón de años en producir, por lo que el posible agotamiento de las reservas existentes es una realidad que no admite discusión.

La posibilidad de agotamiento del petróleo y del gas natural será una realidad en el plazo de 1 ó 2 generaciones.

Las reservas de carbón son menos limitadas (y menos aún si se incluyen los carbones de muy mala calidad). Sin embargo, este combustible es altamente contaminante, de forma que su utilización estará condicionada al desarrollo de tecnologías más limpias en la quema del carbón.

Mucho antes del agotamiento de los recursos convencionales se están produciendo tensiones en los precios del petróleo, ante la falta de capacidad mundial de mantener el ritmo de cre- 
cimiento de la producción que sería necesario para satisfacer la demanda.

\section{Tensiones sociales}

Ya se ha comentado el enorme desequilibrio entre países ricos y pobres en lo que a consumo energético se refiere. Si a ello se añade la concentración de los recursos de combustibles fósiles en unos pocos lugares y que los grandes países productores y los consumidores se sitúan en lados opuestos del planeta, resulta un escenario poco tranquilizador para el equilibrio sociopolítico mundial.

\section{¿Cómo diferenciar potencia de energía?}

La potencia se mide en vatios (W). Se suelen utilizar múltiplos como kilovatios
(kW) -1000 vatios-, megavatios (MW) -1 millón de vatios- o gigavatios (GW) -1000 millones de vatios-. La energia se puede medir en vatios-hora (Wh), o en unidades derivadas, como $\mathrm{kWh}$.

Para entender esta terminología, veamos el siguiente ejemplo: una bombilla de $100 \mathrm{~W}$ tiene una potencia de 100 W siempre, esté encendida o no, pero no consume energía mientras está apagada. Si a lo largo de un día tenemos esa bombilla encendida durante 3 horas, la energía consumida por la bombilla es de $300 \mathrm{Wh} /$ día (100 W x 3 $\mathrm{h}=300 \mathrm{Wh}$ ).

\section{Cuadro $\mathrm{N}^{\circ}$ 2. Unidades de Potencia Eléctrica}

\begin{tabular}{|c|c|}
\hline \multicolumn{2}{|c|}{$\begin{array}{l}\text { CONVERSIÓN DE UNIDADES } \\
\text { ENERGÉTICAS BÁSICAS }\end{array}$} \\
\hline $1 \mathrm{cal}$ & 4,18 julios $(J)$ \\
\hline 1 kWh (kilowatio hora) & $3,6 \mathrm{MJ}$ \\
\hline $1 \mathrm{kWh}$ & $1,36 \mathrm{CV}$ (caballo de vapor) \\
\hline $1 \mathrm{kWh}$ & 3413 BTU \\
\hline 1 TEP (tonelada equivalente de petróleo) & $41,8 \mathrm{GJ}$ \\
\hline 1 TEC (tonelada equivalente de carbón) & 0,7 TEP \\
\hline $1 \mathrm{GWh}$ & 223,3 TEP \\
\hline 1000 barriles de petróleo & 132,05 TEP \\
\hline
\end{tabular}

\section{PRODUCCIÓN DE ENERGÍA ELÉCTRICA}

Para la generación de electricidad a gran escala se recurre a instalaciones denominadas centrales eléctricas, que constituyen el primer escalón del sistema de suministro eléctrico. Dependiendo de la fuente primaria de energía utilizada, las centrales genera- doras se pueden clasificar en los tipos que se citan a continuación.

\section{Térmicas}

En una central térmica se convierte la energía química de un combustible en energía eléctrica. Según el combustible utilizado, se las denomina centrales térmicas de carbón, de fuel o de gas.

Todas las centrales térmicas constan, en su forma más simple, de una 
caldera y de una turbina que mueve un generador eléctrico. La única diferencia entre ellas es el combustible; por tanto, la caldera deberá adaptarse al combustible utilizado. Todos los demás sistemas y componentes son básicamente los mismos.

La caldera es un aparato que sirve para convertir el agua en vapor. El vapor producido, que sale de la caldera, mueve la turbina y, ésta, a su vez, el generador eléctrico. El calor necesario para elevar la temperatura y presión del agua lo proporciona la quema del combustible, ya sea carbón, fuel o gas. El rendimiento de estos sistemas no suele sobrepasar el $33 \%$, desaprovechándose la mayor parte de la energía en pérdidas de calor a lo largo de todo el sistema. A esto habría que añadir las pérdidas en el transporte y distribución de la electricidad a través de las lineas de alta, media y baja tensión. El rendimiento de una central con- vencional en nuestro país, incluyendo distribución hasta los puntos de consumo, estarian alrededor del 25\%.

\section{Ciclo combinado}

En la actualidad, se están construyendo numerosas centrales de las denominadas de ciclo combinado, que son un tipo de central que basa su funcionamiento en el acoplamiento de dos ciclos diferentes de producción de energía, uno con turbina de vapor y otro con turbina de gas. En el ciclo combinado, los gases calientes de escape del ciclo de turbina de gas entregan el calor necesario para mover la turbina de vapor acoplada. Esta configuración permite un empleo más eficiente del combustible. Cada una de estas turbinas está acoplada a su correspondiente generador para producir la electricidad como en una central térmica convencional.

\section{Gráfico $\mathbf{N}^{\circ}$ 3. Central de Generación-CC}

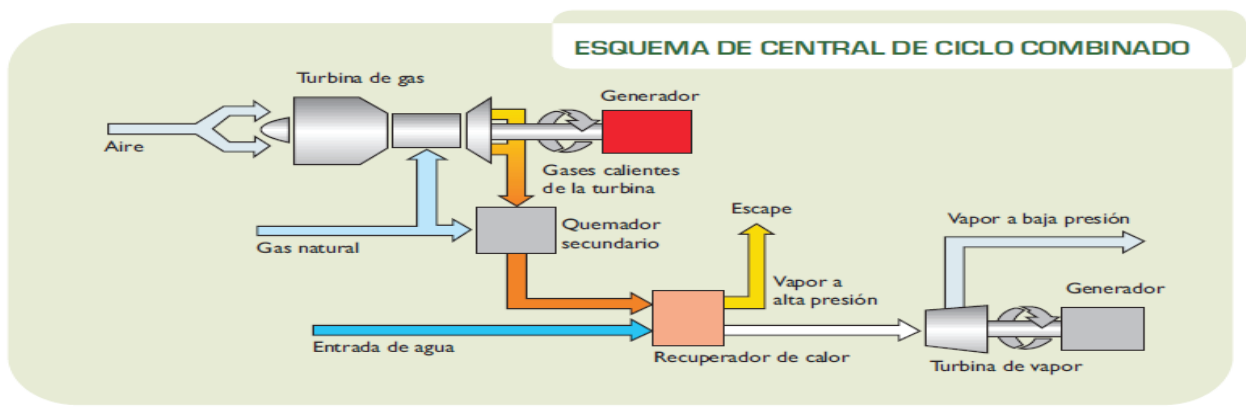

\section{Cogeneración}

Los sistemas de cogeneración son sistemas de producción simultánea de electricidad y calor, partiendo de un único combustible.

El proceso de producción de electricidad es el convencional (ciclo de combustión -turbina- generador eléctrico), pero en el caso de la cogeneración se utilizan los gases de escape (si se emplean turbinas de gas), o el vapor (si se usan turbinas de vapor), que salen a altas temperaturas, para producir calor, el cual se utiliza direc- 
tamente en distintos procesos industriales.

El rendimiento global de este tipo de centrales puede alcanzar el 70\%.

\section{Hidroeléctricas}

Son centrales que generan electricidad mediante el aprovechamiento de la energía potencial del agua embalsada en una presa.

La instalación de centrales hidroeléctricas depende de la posibilidad de construir embalses o presas en los cauces de los ríos para retener el agua, y transformar la energía hidráulica en energía eléctrica. La generación de energía eléctrica se produce al dejar caer el agua desde una cierta altura; esta agua mueve los álabes de una turbina que, a su vez, acciona un generador, produciendo electricidad.

La energía hidráulica posee dos ventajas principales respecto a los combustibles de origen fósil y nuclear: una de ellas es que el agua (que es el combustible) no se consume ni em- peora la calidad, únicamente es explotada; y otra de las ventajas es que no tiene problemas de producción de desechos. Los aprovechamientos mini hidráulicos han permitido el desarrollo de regiones aisladas en todo el mundo.

Estos pequeños proyectos están diseñados para utilizar el caudal de un río o un arroyo mediante la desviación del total o de parte del caudal hacia un canal y, posteriormente, conducirlo a una turbina por medio de una tubería.

\section{Nucleares}

Una central nuclear de fisión, de las que se utilizan en la actualidad, es la que emplea para la generación de energía eléctrica elementos químicos pesados, como el uranio o el plutonio, los cuales, mediante una reacción nuclear, proporcionan calor. Este calor se emplea para producir vapor y, a partir de este punto, el resto de los procesos en la central son análogos a los de una central térmica convencional.

\section{Gráfico $\mathrm{N}^{\circ}$ 4. Central de Generación $\mathrm{CN}$}

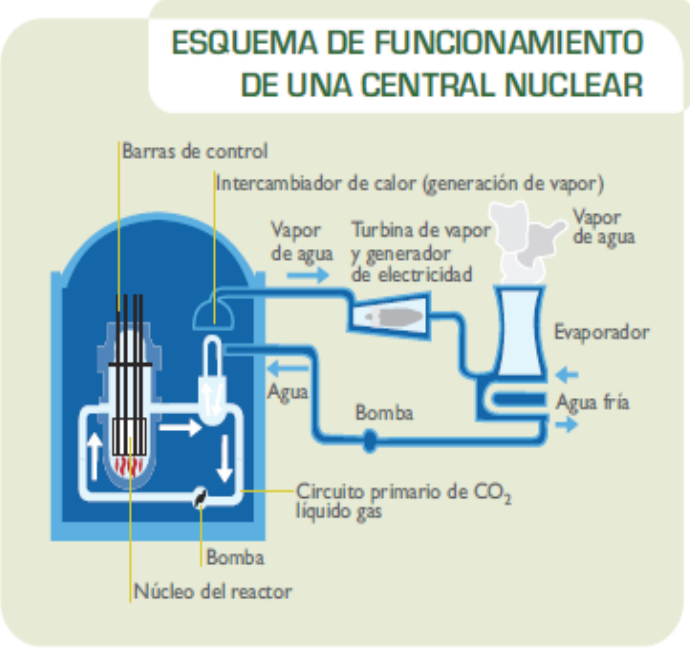




\section{Centrales de energias renovables Parques eólicos}

Conjunto de aerogeneradores que se ha popularizado en los últimos años debido a que la energía eólica se considera una "energía limpia" (respetuosa con el medioambiente), ya que no requiere una combustión que produzca residuos contaminantes ni provoca la destrucción de recursos naturales.

No obstante, la cantidad de energía producida de origen eólico es aún una minima parte de la que se consume por los países desarrollados y del potencial que representa.

\section{Centrales solares fotovoltaicas}

Los paneles solares fotovoltaicos transforman la radiación solar direc- tamente en electricidad. Las plantas o centrales solares fotovoltaicas están constituidas por una serie de paneles fotovoltaicos conectados en serie y/o en paralelo, que vierten la electricidad producida a la red eléctrica.

En la actualidad, en casi toda Europa se está fomentando la construcción de este tipo de centrales a través de incentivos económicos.

\section{Centrales solares térmicas de alta temperatura}

Utilizan el calor de la radiación solar para calentar un fluido y producir vapor para mover un generador, como en una central térmica convencional, pero en la que el combustible es el Sol.

\section{Gráfico $\mathbf{N}^{\circ}$ 5. Centrales Solares}
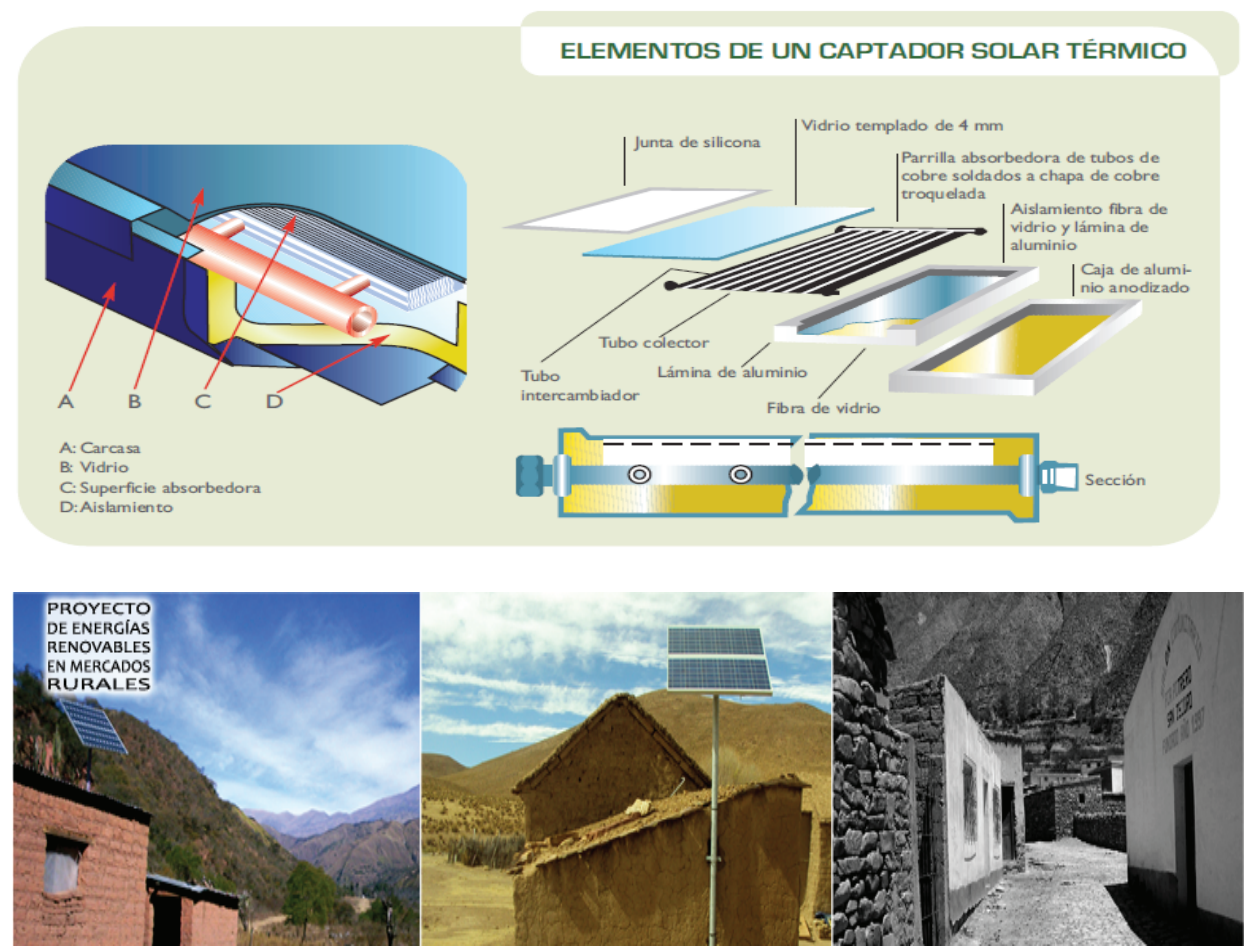


\section{Centrales marinas}

Dentro de las centrales que se instalan en el mar, podemos distinguir: las centrales maremotrices, las de olas y las que aprovechan las corrientes marinas. Las centrales maremotrices aprovechan los cambios de altura de las mareas para mover las turbinas, mientras que las de olas utilizan el movimiento de éstas con el mismo fin.

\section{Centrales geotérmicas}

La energía geotérmica es la que procede del calor interno de la Tierra. Existe una gran diferencia entre la temperatura de la superficie terrestre y la de su interior.

El calor concentrado en el interior tiende a escapar de forma natural, como ocurre en las fuentes hidrotermales o en los géiseres. También se puede extraer a partir de perforaciones en yacimientos localizados en el subsuelo.

\section{Centrales minihidráulicas}

Son las centrales hidráulicas cuya potencia es de $10 \mathrm{MW}$ o menos. En el aspecto medioambiental, todas ellas poseen las ventajas de las energías renovables (energía limpia, autóctona e imperecedera), aunque también presentan una serie de posibles inconvenientes, según el tipo de renovable de que se trate.

La geotérmica, frente al resto de las renovables, presenta la gran ven- taja de producir electricidad de forma constante (en este sentido funciona casi como una central convencional), a diferencia de las otras centrales mencionadas, que dependen de la disponibilidad del viento, sol, etc.

Todas estas centrales, excepto las fotovoltaicas, tienen en común el elemento generador en sí, que no es otro que un generador eléctrico, movido mediante una turbina, que será distinta dependiendo del tipo de energía primaria que se utilice.

A pesar de todos los tipos de centrales renovables indicadas, la mayor parte de la energía eléctrica generada proviene de los tres primeros tipos de centrales: térmica convencional, hidráulica y nuclear.

\section{La biomasa}

La energía del Sol es utilizada por las plantas para sintetizar la materia orgánica mediante el proceso de fotosintesis. Esta materia orgánica puede ser incorporada y transformada por los animales y por el hombre. El término biomasa abarca un conjunto muy heterogéneo y variado de materia orgánica y se emplea para denominar a una fuente de energía basada en la transformación de la materia orgánica utilizando, normalmente, un proceso de combustión. 


\section{Gráfico $N^{\circ}$ 6. Ciclo de la Biomasa}

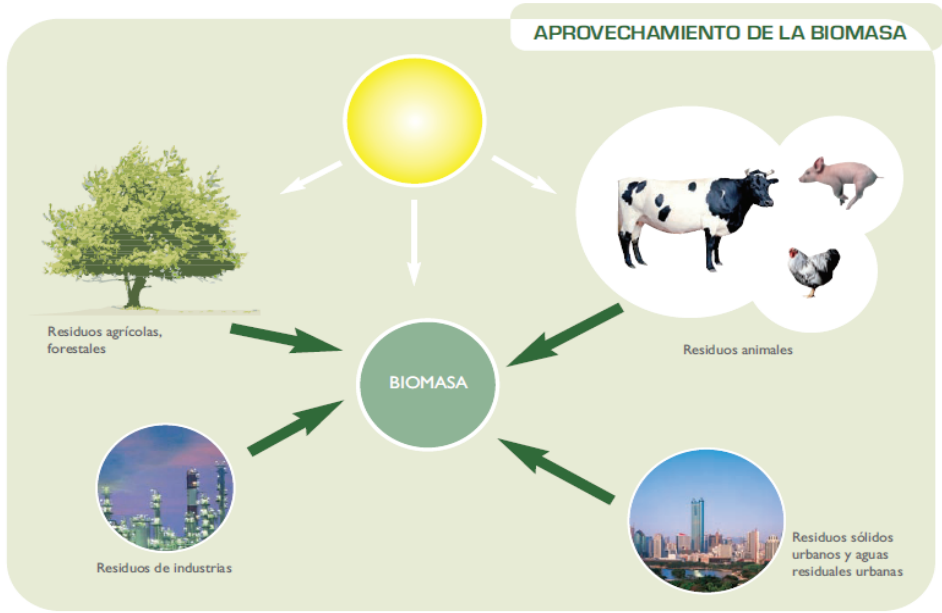

\section{Cuadro $N^{\circ}$ 3. Energias Renovables En Matriz Energética en el Perú}

\begin{tabular}{|l|l|l|}
\hline OPCIÓN & PLAN BASE & PLAN NUMES \\
\hline $\begin{array}{l}\text { Estructura de generación } \\
\text { eléctrica }\end{array}$ & $\begin{array}{l}\text { Hidroeléctrica 70\%, } \\
\text { Gas Natural 25\%, RER 5\% }\end{array}$ & $\begin{array}{l}\text { Hidroeléctrica 40\%, } \\
\text { Gas Natural 40\%, RER 20\% }\end{array}$ \\
\hline Petroquímica & Región Ica, Sur & Base \\
\hline Transporte de Gas & Sur y Norte & Base \\
\hline Exportación & Contratos vigentes & Base GN; EE Exportación regional \\
\hline Petróleo & No se Desarrollan crudos pesados & Desarrollo de crudos pesados \\
\hline Biocombustibles & $5 \%$ diesel, 7.8\% etanol & $5 \%$ diesel, 10\% etanol \\
\hline Cobertura de Gas & $14 \%$ al 2040 & $18 \%$ al 2040, máxima cobertura \\
\hline Exploración GN & 3 a 4 TCF adicionales por quinquenio & Base \\
\hline Eficiencia Energética & Situación actual & $15 \%$ de ahorros \\
\hline
\end{tabular}

Con esta opción se alcanzan en el año 2040 los objetivos de política energética

Cuadro $N^{\circ}$ 4. Proyectos de Recursos Energéticos Renovables (RER)

\begin{tabular}{|l|l|c|c|c|}
\hline \multicolumn{1}{|c|}{ Proyecto } & \multicolumn{1}{|c|}{ Tecnología } & $\begin{array}{c}\text { En } \\
\text { operación }\end{array}$ & MW & $\begin{array}{c}\text { Inversiones } \\
\text { Estimadas } \\
\text { (MM US\$) }\end{array}$ \\
\hline Cupisnique & Eólica & 2012 & 80 & 242,4 \\
\hline Talara & Eólica & 2012 & 30 & 101,2 \\
\hline Marcona & Eólica & 2012 & 32 & 43,6 \\
\hline Panamericana & Solar FV & 2012 & 20 & 87,0 \\
\hline Majes & Solar FV & 2012 & 20 & 70,5 \\
\hline Repartición & Solar FV & 2012 & 20 & 70,2 \\
\hline Tacna & Solar FV & 2012 & 20 & 85,0 \\
\hline Paramonga & Biomasa con R.A. & 2010 & 23 & 31,0 \\
\hline Huaycoloro & Biomasa con R.U. & 2011 & 4,4 & 10,5 \\
\hline 17 centrales & Hidroeléctrica & 2013 & 179,7 & 285,1 \\
\hline Total & & & 429,1 & 1026,5 \\
\hline Fuentes: MINEM y OSINERGMIN & & & \\
\hline
\end{tabular}




\section{¿Qué es la Operación del SEIN?}

Consiste en efectuar la programación y coordinación de la operación integrada de los recursos de generación y transmisión del SEIN, de acuerdo con lo estipulado en la Ley de Concesiones Eléctricas (LCE) y la Ley para asegurar el desarrollo Eficiente de la Generación Eléctrica (Ley 28832), cumpliendo con sus respectivos reglamentos y los procedimientos técnicos aprobados por el OSINERGMIN.

\section{¿Qué es el COES?}

El Comité de Operación Económica del Sistema (COES), es el organismo que opera el sistema eléctrico peruano, administra el mercado eléctrico y planifica la transmisión eléctrica del sistema con criterios de economía, calidad y seguridad. El COES es una entidad privada sin fines de lucro, cuyos aportantes son los agentes del mercado.

\section{¿Quiénes son integrantes del COES?}

Son las empresas de generación de electricidad, transmisión eléctrica, distribución eléctrica y los usuarios libres (grandes consumidores), quienes forman parte del COES.

\section{Gráfico $\mathbf{N}^{\circ}$ 7. Sistema Interconectado Nacional}

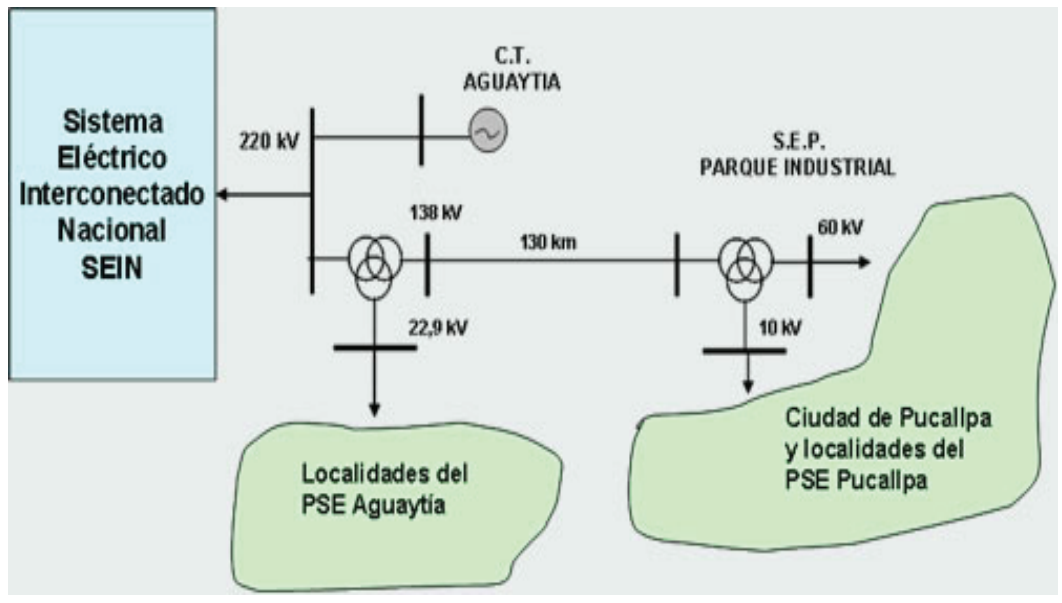

\section{GENERACIÓN DISTRIBUIDA (GD)}

La generación distribuida, es la generación in situ. Generación embebida, generación descentralizada,generación dispersao energía distribuida, consiste básicamente en la generación de energía eléctrica por medio de muchas pequeñas fuentes de energía.
La definición más global de la generación distribuida vendría a decir que es aquella que se conecta a la red de distribución de energía eléctrica y que se caracteriza por encontrarse instalada en puntos cercanos al consumo. 


\section{Gráfico $\mathbf{N}^{\circ}$ 8. GD- Integración de las Energias Renovables con el Sistema Eléctrico}

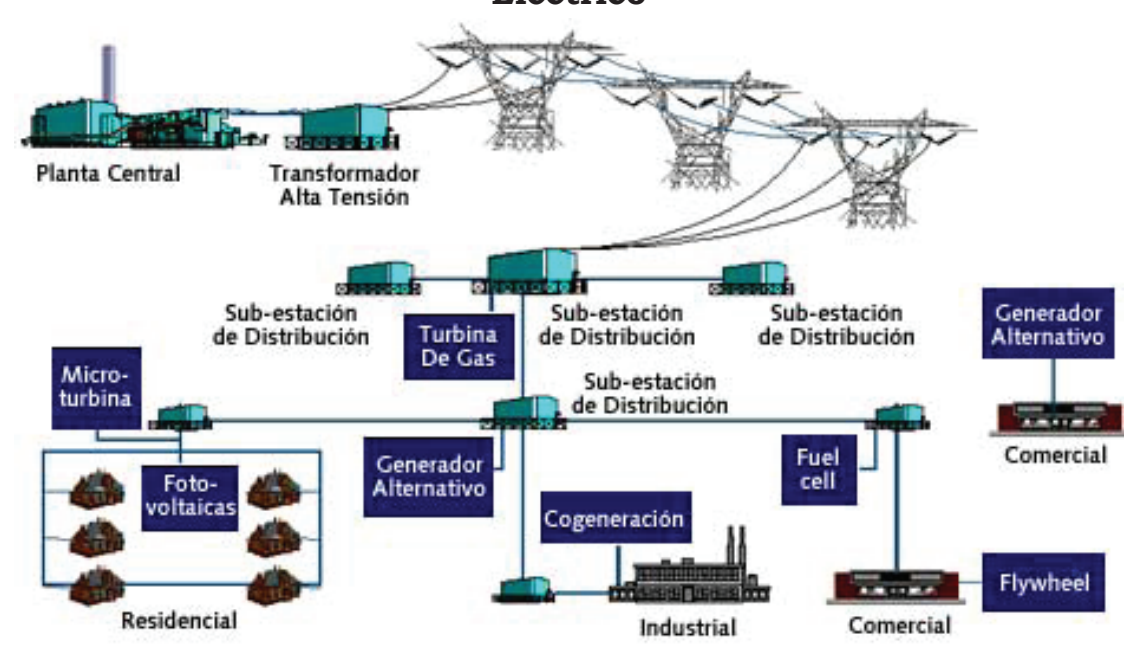

Los sistemas empleados como la distribución eléctrica, como de las fuentes de energía distribuida (FED), comunicaciones. En este sentido, la son plantas de generación de energía distribución eléctrica es el control de a pequeña escala entre el rango de la generación distribuida, la minimi$3 \mathrm{~kW}$ a $10 \mathrm{~kW}$, usadas para proporcionar una alternativa o una ayuda a las zación de las pérdidas o la mejora de la gestión de activos y las comunicatradicionales centrales de generación eléctrica.

Utilizando protocolos y sistemas ciones, es la integración segura de distintas tecnologías, la autogestión de la redundancia de caminos y la mejora estándares y abiertos, se han implecontinua de las prestaciones de la red.

mentado tanto funciones propias de

\section{Gráfico $\mathbf{N}^{\circ}$ 9. Evolución de la Red Eléctrica}

RED ACTUAL

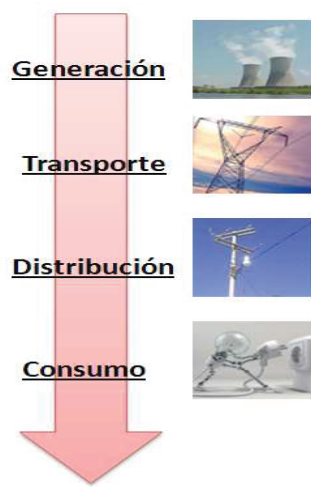

RED FUTURO

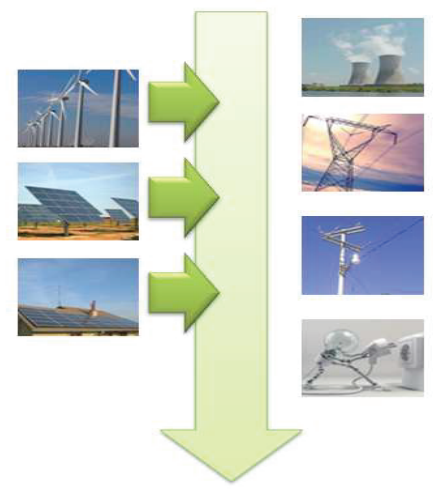




\section{Gráfico $\mathrm{N}^{\circ}$ 10. Comparación de Distribución de Energía Tradicional y Energia Distribuida}

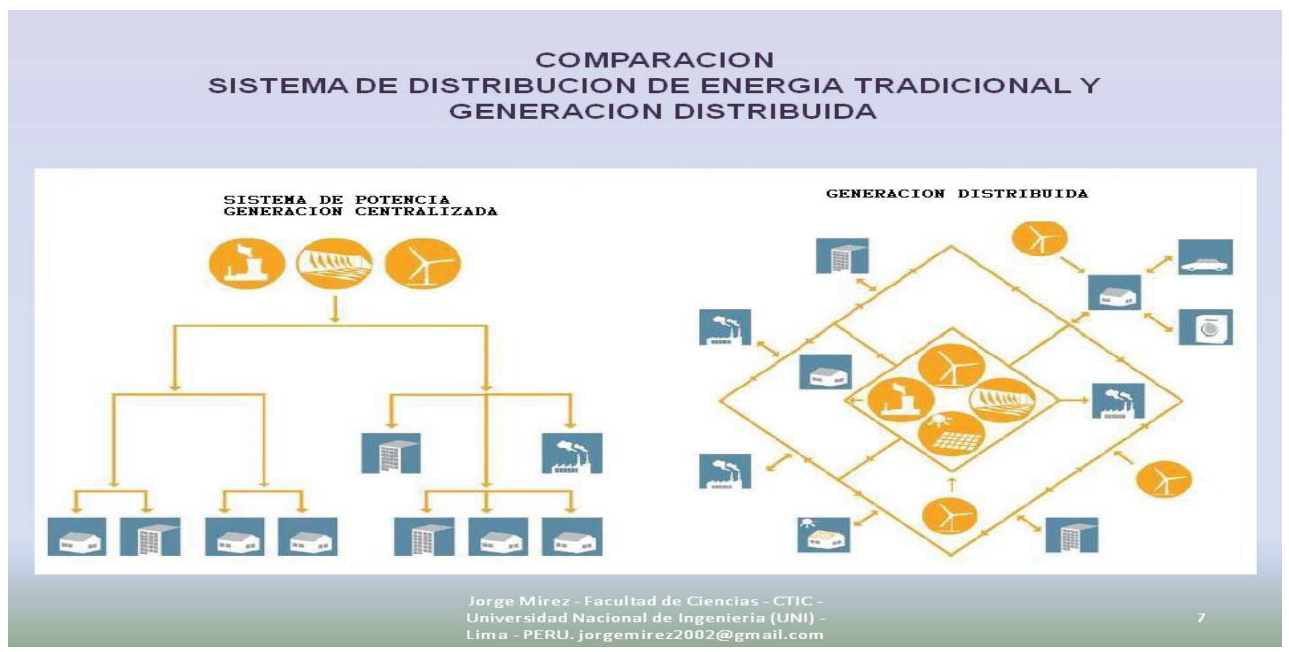

\section{CONSIDERACIONES EXPERIMENTALES}

Para el inicio del estudio, es de vital importancia familiarizarse con el siste- ma eléctrico de potencia, en el sentido de que las energias renovables pasarán a ser parte de este sistema, considerando que estas se integrarán como elemento importante en el sistema.

\section{Gráfico $N^{\circ}$ 11. Sistema Eléctrico de Potencia}
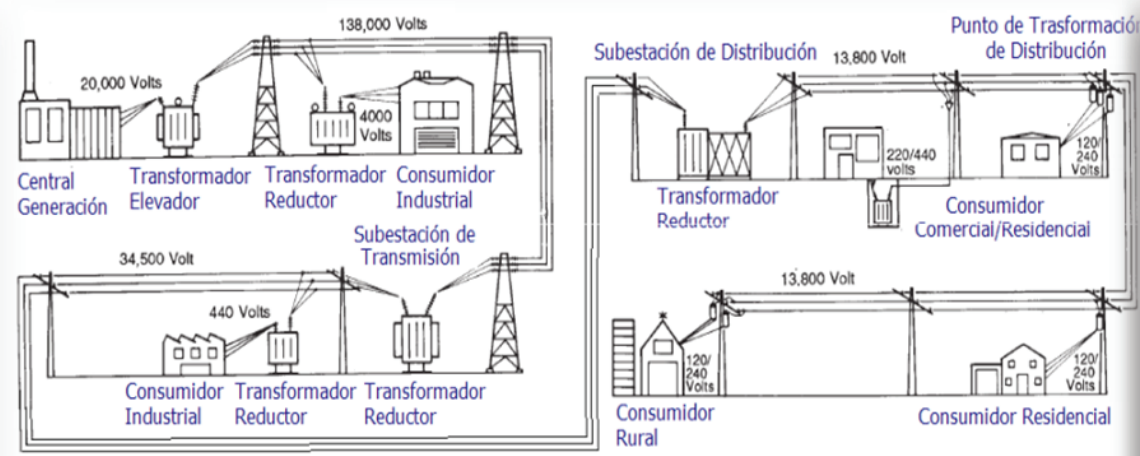

Circuito eléctrico a considerar para definir las variables eléctricas que se deben manipular: 


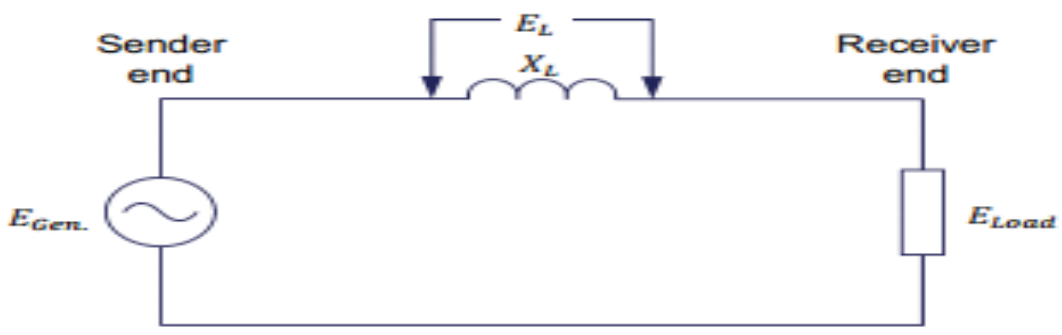

Se le denomina circuito equivalente simplificado de una línea de transferencia de potencia de transmisión de corriente alterna, a partir de una estación de generación (lado del emisor), a una estación de distribución (lado del receptor).

Desde La arquitectura desarrollada, es capaz de extender a cualquier punto de la red de baja tensión, de manera distribuida y eficiente, toda la capacidad de monitorización y control que hasta la fecha se tenía únicamente en la red de alta tensión. Utilizan- do protocolos y sistemas estándares y abiertos, se han implementado tanto en funciones propias de la distribución eléctrica, como de las comunicaciones.

Tomando en consideración un sistema eléctrico de potencia eficiente propuesto por el SEIN, como se muestra en el diagrama unifilar de una red de 5 barras, se aplicó el simulador PowerWorld para determinar las variables en las barras de generación en MVA, datos que nos servirán para calcular la cantidad de MVA que se pueda obtener de las energías renovables.

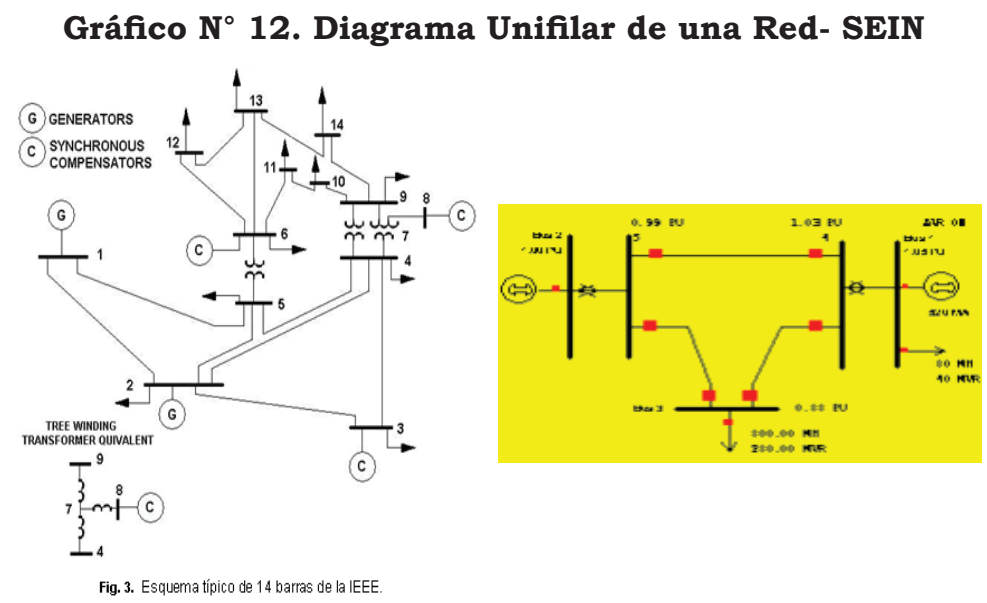

Es importante indicar que además de la generación, el programa nos permite conocer las variables de las lineas de transmisión, lo cual nos servirá para determinar los beneficios que al respecto se obtendrán de las energías renovables, al estar en el mismo lugar de los consumidores, dando origen a la Generación Distribuida.

Interconexión: Determinado el tipo de generación de energia renovable a 


\section{considerar se deberá tener en cuenta como interconectar al sistema}

Por lo tanto, conocidas las variables de la generación, un aspecto necesario en la GD es la interconexión con la red eléctrica, para poder cubrir cualquier eventualidad del sistema de compra o venta de energía eléctrica. Algunos de los aspectos técnicos a considerar en la interconexión, son:

- Relevadores de protección.

- Conexión del transformador.

- Sistema de puesta a tierra.
- Coordinación de protecciones y regulación de la tensión de la compañía.

- Equipos de calidad de servicio.

- Conformidad con normas de los convertidores de potencia.

- Monitoreo y control remoto del grupo.

- Mantenimiento preventivo y correctivo periódico.

- Sistema de comunicación entre el operador privado y el controlador de la red de distribución.

\section{Gráfico $\mathbf{N}^{\circ}$ 13. Esquemas planteados para la interconexión}
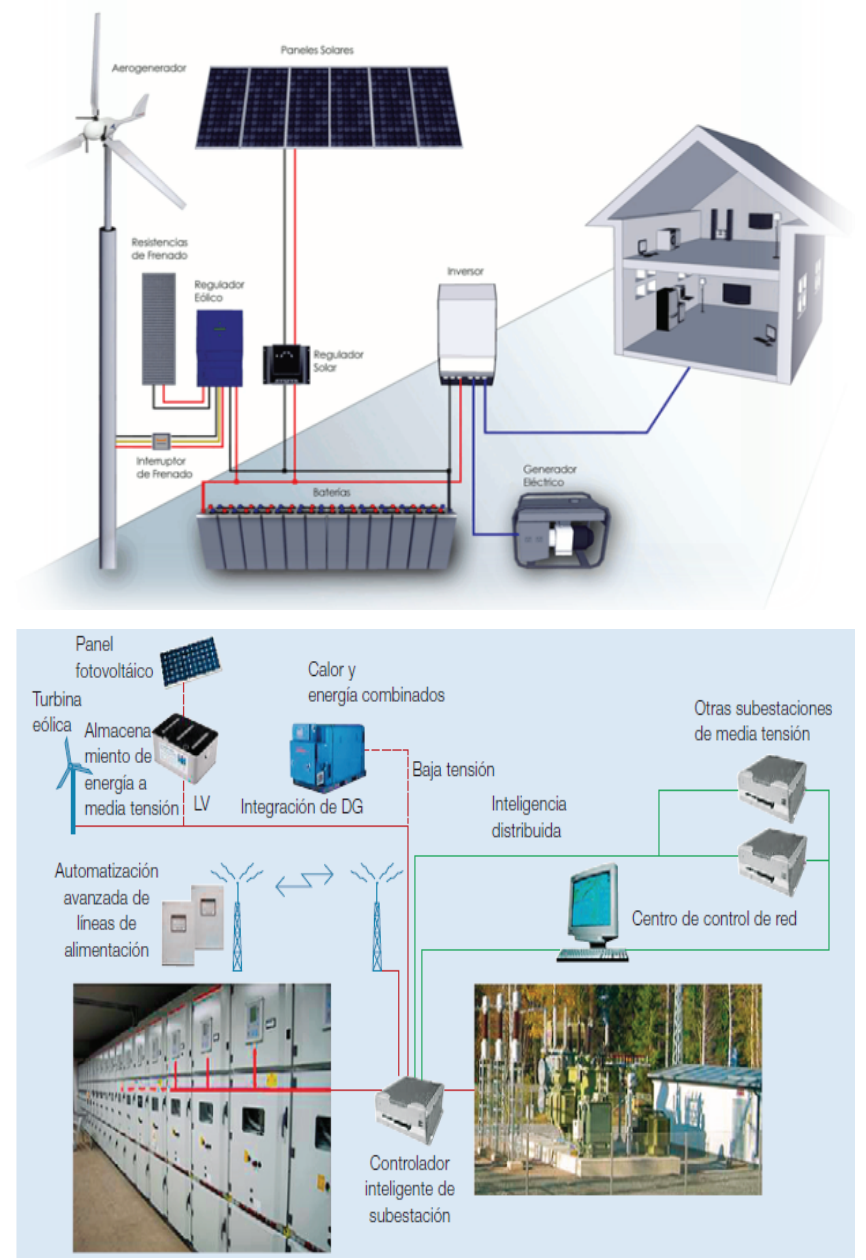
Se determinó que la GD tiene como parte fundamental la Automatización de subestaciones, las que necesitan sistemas de Protecciones integradas a fin de evitar las perturbaciones en los armónicos que genera la AC. Estos sistemas pueden ser:

RAT (Regulación Automática de Tensión)

TAC (Transferencia Automática de Cargas)

Comunicación e inter-operatividad de dispositivos bajo el estándar IEC 61850.

Para poder determinar que tipo de generación es factible implementar en la zona de estudio, fue necesario plantearse lo siguiente:

¿Se desea producir calor a efecto de reducir el consumo de combustibles fósiles caros para la calefacción?

Entonces, un sistema de biomasa podría ser el indicado. Asimismo, es posible que se pueda incorporar calefacción solar del agua al lado de un sistema fotovoltaico.

$¿$ El objetivo principal es dotar de electricidad a un emplazamiento rural?

Entonces, un sistema hidroeléctrico, eólico o fotovoltaico de pequeña escala, podría ser la mejor opción. Es más complejo producir electricidad a partir de biomasa que con otros sistemas; además, las opciones de tecnología todavía están muy limitadas.

\section{Disponibilidad y tipo de recursos en la zona de estudio}

A continuación, se investigó si cuenta con recursos de energía renovable; por ejemplo:
¿Es una región de fuertes vientos? ¿Tienen acceso a recursos forestales $\mathrm{u}$ otro abundante de biomasa que se pueda utilizar? ¿Hay un río o arroyo que se pueda aprovechar para generar electricidad?

Otro aspecto importante a considerar fue como integrar estas energías al sistema eléctrico, dando lugar a la Generación Distribuida. El planteamiento fue el siguiente:

¿Cómo puede vender la electricidad producida? ¿Existe una estipulación sobre medición neta en el área?

Al respecto, las políticas en nuestro país son bastante incipientes, sin embargo con la implantación de los Smart Meter y la Smart Grid, se está desarrollando la Generación Distribuida, con el nuevo concepto del PROSUMIDOR (productor-consumidor de energía)

Otra posibilidad contemplada en la producción de energía se consideró la cogeneración, que en muchos casos satisfacen al 100\% los requerimientos térmicos de los establecimientos industriales o comerciales, en los que se instalan y permiten ahorros de energia primaria de $30-35 \%$ respecto al consumo anterior al desarrollo del proyecto. Alcanzan eficiencias de aprovechamiento de la energía primaria superiores al 70\% y, además, proporcionan energía eléctrica excedente que puede ser entregada a la red o ser consumida en otras instalaciones asociadas al sistema de Cogeneración.

Por lo tanto, se consideró una ventaja utilizar la cogeneración, ya que tiene una eficiencia energética que 
aprovecha tanto el calor como la ener- eléctrica convencional y para las necegía mecánica o eléctrica de un único sidades de calor una caldera convenproceso, en vez de utilizar una central cional.

\section{Gráfico $\mathbf{N}^{\circ}$ 14.- Esquemas considerados para cogeneración GENERACIÓN MEDIANTE UNA PLANTA DE COGENERACIÓN}

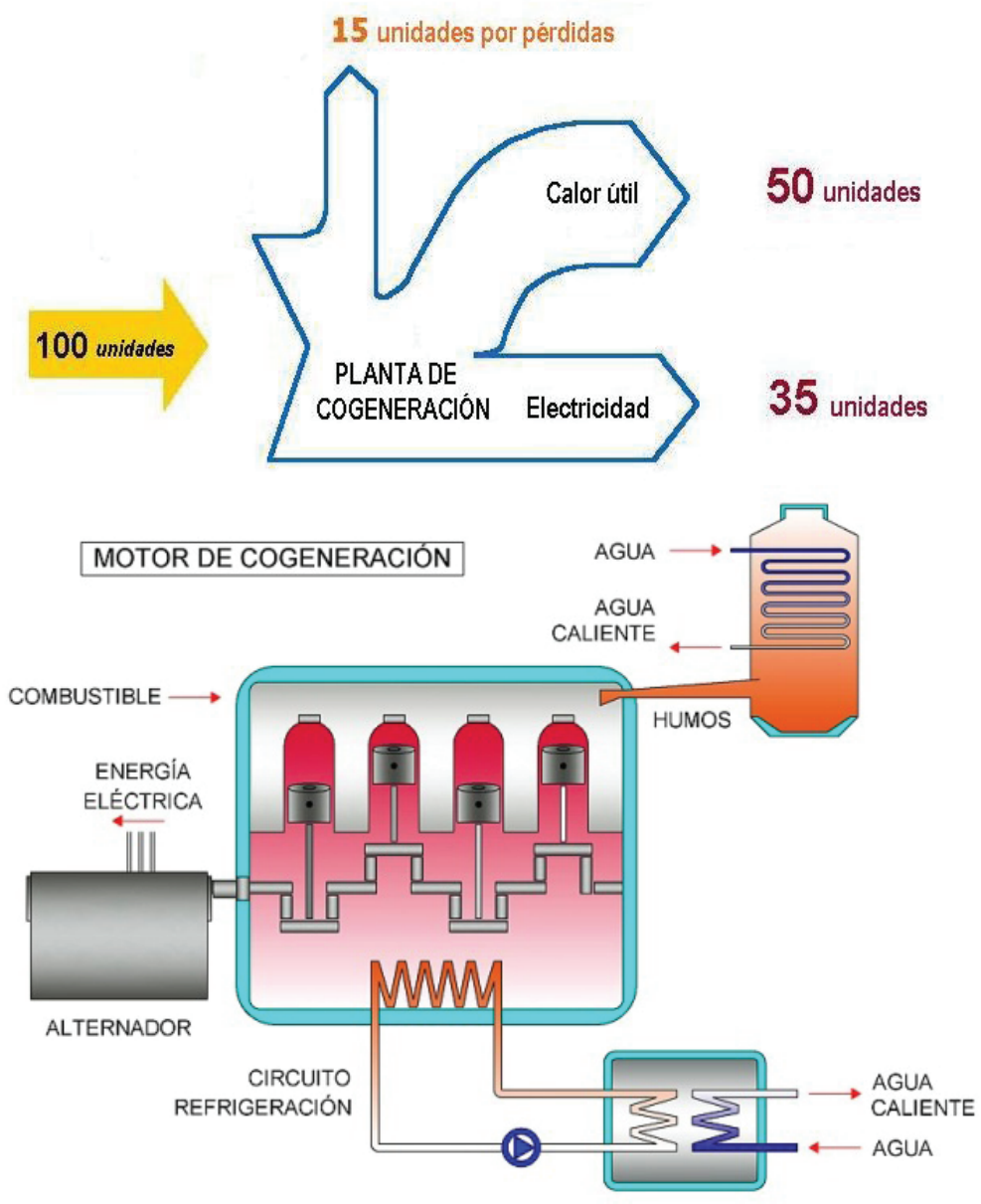

Almacenamiento de Energia: Las energías renovables producen energía en DC, la cual debe ser acumulada para su posterior inversión en AC para su utilización. Al respecto, se investigaron una serie de tecnologías que cumplen con esta función, tales como: 


\section{Gráfico $N^{\circ}$ 15. Sistemas de almacenamiento SMES (Se almacena energía en campo magnético)}

\section{STANDBY MODE}

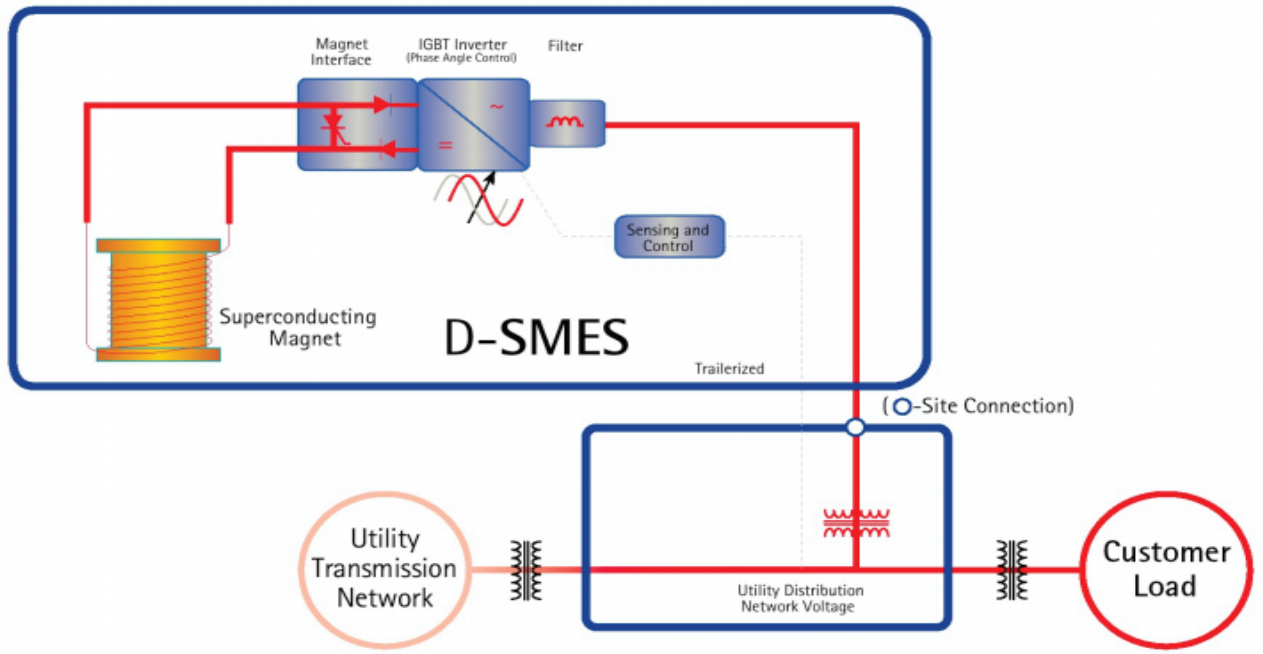

Sistemas con baterias (UPS): el problema de las baterías es su coste, su volumen, sus ciclos de trabajo y su vida útil. Su capacidad de almacenamiento va de 10 a 60 minutos; su utilidad típica esta en los ordenadores para reducir y limitar los picos de demanda, mejorar la calidad y la falibilidad del suministro; los últimos desarrollo en esta tecnología, son las pilas de Li-ion.

Dentro de las tecnologias de almacenamiento "eléctrico", se tienen:

Baterias: el más extendido, Super condensadores

Volantes de Inercia mecánica (Flywheels)

SMES - superconductor de almacenamiento de energía magnética

CAES: almacenamiento de energía por aire comprimido

\section{CONCLUSIONES}

Después del 2016, en nuestro país no se cuenta en cartera la inclusión de nuevos proyectos de generación y, si se considera los prolongados tiempos de ejecución de los proyectos por los diferentes permisos necesarios (licencia social y/o trámites burocráticos), la vulnerabilidad del sistema se incrementaría con los costos que ello acarrea. En tal sentido, según el análisis elaborado por el COES, se proyecta que para el 2018 los costos marginales promedio ponderado, se ubicarían alrededor de los 300 US\$/MW.h, siendo superior a los precios registrados en el 2008.

Por ello, se considera necesario implementar medidas con visión en el largo plazo en proyectos de energía renovable, y no centrarse en soluciones cortoplacistas, con el objetivo de 
garantizar la confiabilidad del SEIN a fin de evitar los costos resultantes de una planificación inadecuada.

Es importante tener en cuenta que en la generación eléctrica convencional, las plantas se localizan lejos de los centros de consumo, por lo que existen de un $4 \%$ a un $19 \%$ de pérdidas de transmisión, distribución y robo, dependiendo de la tensión de la red y de la región tarifaria del país.

Se ha logrado determinar que con la Cogeneración, estas pérdidas disminuyen o se eliminan, por estar localizadas en los centros de consumo. La Cogeneración, al consumir menos combustible, reduce las emisiones contaminantes. La reducción depende de la tecnología, el factor de planta y el tipo y calidad del combustible.

De esta manera, al tener mayor eficiencia de conversión y reducir el consumo de energía primaria, los costos de energía eléctrica de las plantas de Cogeneración son menores que las tarifas de la red de servicio público.

Consideramos que esto puede ser aplicable a la mayoría de los sectores industriales, la agroindustria y en el sector servicios, como hoteles, hospitales, centros comerciales, centros deportivos y escuelas.

Por consiguiente, se determinó que se logra reducir costos incrementando la competitividad, además de reducir el riesgo de cortes del servicio eléctrico por contar con la planta de Cogeneración y el respaldo de la red pública.

La Cogeneración se instala en los centros de carga. Esto implica una descentralización de la generación de la energía, lo que permite desarrollos regionales y la creación de empleos. Un proyecto de cogeneración involucra y brinda beneficios a alrededor de 30 empresas relacionadas al mantenimiento y suministro de materiales para mantener y asegurar una óptima operación de la instalación.

Este aspecto es fundamental, con base en las nuevas normativas que se han dictado, nuevas tecnologias que aparecen desde protocolos de comunicación hasta equipos para telecomando y supervisión de los distintos componentes de la red, nuevas protecciones, nuevos medidores, nuevos sistemas de gestión de la demanda, etc. lo que hace que los profesionales de la actividad nos veamos obligados a una actualización permanente para poder hacer frente a los nuevos desafios que llevarán aparejadas las futuras Smart Grids y la Generación Distribuida (GD).

Para lograr la ejecución de estos proyectos, se debe contemplar la menor interferencia politica, con el fin de evitar desequilibrios innecesarios en el sistema o en los diferentes agentes que participan del sistema. No menos importante es la necesidad de tomar acciones concretas e inmediatas para atraer y acelerar las inversiones, pues existen los recursos y capacidades necesarias para llevar a cabo dichos proyectos. Asimismo, se deben elevar los estándares de gobierno corporativo de las entidades estatales que participan del SEIN, así como las eficiencias tanto operativas como comerciales que son ampliamente superiores por las empresas privadas. 


\section{REFERENCIAS BIBLIOGRÁFICAS}

Mendoza, Jaime R. (2012). "Marco Legal de las Energías Renovables en el Perú".

CONAE Generación Distribuida. (2005). "Comisión Nacional para el ahorro de energía. México D.F."

Equilibrium Clasificadora de Riesgo S.A (2013). "Análisis del Sector Eléctrico Peruano". Lima.

COES (2014). "Informe de Diagnostico de las Condiciones Operativas del SEIN 2015 - 2024”. Lima.

Silftung, Friedrich Ebert (2012). Matriz Energética en el Perú y Energías Renovables - Barreras para el Desarrollo. Lima.

Grupo de Representantes de la Subcomisión para Promover Proyectos de Cogeneración (2004). "Iniciativa para el Fomento de la Cogeneración. Hacia una política de conversión eficiente de la energía. Lima".

Graficas Elisa S.A (2007). Guía básica de generación distribuida - fundación de la energía de la comunidad de Madrid. España.

\section{WEB-GRAFÍA}

Informe ASIF. Hacia una electricidad respetuosa con el medio ambiente. Encontrado el 15-05-2014, en www.asif.org

Pontificia Universidad Católica del Perú: Biomasa. Encontrado el 10-6-2014, en http// www.puc.edu.pe/invest/grupo/energias/powerbio.ppt

Portal del medio ambiente: www.ambientum.com 